\title{
Nanocrystalline CVD Diamond Coatings on Fused Silica Optical Fibres: Optical Properties Study
}

\author{
M. FICEK ${ }^{a, *}$, R. BOGDANOWICZ ${ }^{a}$ AND J. RYL ${ }^{b}$ \\ ${ }^{a}$ Department of Optoelectronics and Electronic Systems, Faculty of Electronics, Telecommunications and \\ Informatics, Gdańsk University of Technology, G. Narutowicza 11/12, 80-233 Gdańsk, Poland \\ ${ }^{b}$ Department of Electrochemistry, Faculty of Chemistry, Corrosion and Material Engineering, \\ Gdańsk University of Technology, G. Narutowicza 11/12, 80-233 Gdańsk, Poland
}

(Received May 12, 2014; revised version January 19, 2015; in final form January 20, 2015)

\begin{abstract}
Seeding and growth processes of thin diamond films on fused silica optical fibres have been investigated. Glass pre-treatment by dip coating in two detonation nanodiamond (DND) seeding media has been studied. The DND suspension in ethyl alcohol and dispersion of DND in dimethyl sulfoxide (DMSO) with polyvinyl alcohol (PVA) were chosen for the seeding purpose. The grain size distribution of nanodiamond particles in both seeding media was kept at the same level (approximately $10-50 \mathrm{~nm}$ ). After the seeding nanocrystalline diamond films were deposited on the fibres using microwave plasma assisted chemical vapour deposition system. The results of the process were investigated using numerical analysis of scanning electron microscopy images. The molecular structure of diamond has been examined with micro-Raman spectroscopy. Thickness, roughness and optical properties of the nanocrystalline diamond films in VIS-NIR wavelength range were investigated on reference samples using spectroscopic ellipsometry. Light reflection at the fibre end-face for different deposition parameters was also investigated. Proposed seeding method can be further effectively applied for manufacturing of optical fibre sensors. Due to extraordinary properties of diamond, which include high chemical and mechanical resistance, such films are highly desired for optical sensing purposes.
\end{abstract}

DOI: 10.12693/APhysPolA.127.868

PACS: 81.15.Gh, 81.05.ug, 82.80.Gk, 68.55.A-, 78.66.Qn

\section{Introduction}

Optical fibres have been extensively used for developing a number of sensing devices $[1,2]$. Numerous optical fibre sensors have been devised for the measurement of physical parameters like temperature [3], pressure [4], or displacement [5].

Optical fibres designed and fabricated mainly for telecommunication systems are coated with polyester coatings which protects the fibre against mechanical damages and harsh environmental conditions [6]. However, the coating does not protect the fibre well against some acids [7].

Carbon layers are well known as a passivation coating for many electronic devices [8]. Diamond films show a combination of properties which makes them attractive also for sensing devices. The properties include, e.g., optical transparency in broad wavelength range $[9,10]$, chemical stability [11], high thermal conductivity [12] and biocompatibility $[13,14]$. Diamond is also known from its good mechanical properties [15]. Thin diamond films can protect optical fibres from either mechanical damage or chemically harsh environment. Thanks to optical properties of the diamond films, which include high refractive index, the films can be also applied for enhancing sensing properties of optical fibre devices [16].

${ }^{*}$ corresponding author; e-mail: mateuszficek@gmail.com

Seeding is a key parameter that affects strongly diamond films growth on various substrates [17-19] and can be performed with a number of methods [20]. The most commonly methods of seeding include treating ultrasonically with diamond slurry [21,22], coating substrates with carbon materials [23] or using the interlayer containing nanodiamond $[24,25]$. Low seeding efficiency has negative effect on diamond films like: discontinuous films, cracks, partial covering of substrates, large thickness gradient or even lack of film growth [26]. On the other hand, it is quite difficult to achieve good quality diamond-fibre interface due to large difference of properties and composition between optical fibres/fused silica materials [27, 28]. May et al. [29] showed that it is possible to deposit highly resistive diamond on optical fibres. Rabeau et al. [30] have shown nitrogen doped diamond films deposited on optical fibre end-face for fluorescence waveguiding. Our previous work shows that application of high-power sonication seeding enables to obtain diamond film in different suspension (water/DMSO). However, the high-power can erode/cavitate optical fibres [31]. Dip-coating seeding seems to be promising method for seeding of optical fibres. This method does not include any treatment that could damage optical fibre. Scorsone et al. proposed seeding with PVA which contained nanodiamond particles. PVA was chosen for its excellent film making properties, high viscosity and high solubility in water and various organic solvents or in water and other various media [32, 33].

In our study, we proposed to use two different types of suspension with diamond nanoparticles. The first 
proposed suspension is PVA with diamond slurry based on DMSO solvent. To the best of author's knowledge the use of PVA/DMSO suspension for optical dip-coating fibre seeding has never been reported before. The second proposed is an ethanol suspension with nanodiamond. Dispersed particles in both suspensions reach high concentration and low diameter of diamond particles 4$5 \mathrm{~nm}[21,22]$. Since optical fibres have cylindrical shape we had to adopt dip-coating method instead of typically used spin coating.

In this paper, we discuss results of dip-coating seeding in two different suspensions and lately growth of diamond by chemical vapour deposition (CVD) on fused silica optical fibres.

Samples deposited on optical fibres were used for light transmission measurements, to test if process of nucleation either CVD diamond growth do not damage waveguiding properties of optical fibre. Substrate temperature have the crucial impact influence on diamond films synthesis process and kinetics of pyrolysis reaction on the growth surface $[34,35]$. Diamond growth process was performed by microwave plasma assisted chemical vapour deposition (MW PA CVD). Scanning electron microscopy (SEM) was enhanced to investigate morphology of nanocrystalline diamond films. Molecular structure was examined by micro-Raman spectroscopy. The growth rate of the films, thickness and their optical properties in VIS-NIR wavelength i.e. refractive index and extinction coefficient were estimated using spectroscopic ellipsometry (SE).

\section{Experimental}

\subsection{Dip-coating seeding}

In order to investigate influence of dip-coating seeding on diamond growth, we prepared a single mode optical fibre (cleaved Corning SMF28, cladding diameter of $125 \mu \mathrm{m}$, approximately $5 \mathrm{~cm}$ in length) whose polymer coating was mechanically removed. The investigated optical fibre is made of fused silica cladding, which is high quality amorphous $\mathrm{SiO}_{2}$, and germanium-doped fused silica core (diameter of $8.2 \mu \mathrm{m}$ ). A set of $p$-type (100) silicon wafers was used as a reference samples. The fibres and silicon wafers were cleaned in the ultrasonic bath containing acetone for $5 \mathrm{~min}$, then rinsed in 2-isopropanol and nitrogen dried.

Then, the optical fibres have undergone hydrogenation. Hydrogenation was performed in microwave $\mathrm{H}_{2}$ plasma at $1300 \mathrm{~W}$ by $30 \mathrm{~min}$. During the process total flow of gas reaching $300 \mathrm{sccm}$ and pressure was kept at 50 Torr. Hydrogenation removes polyimide, which hinders the growth of the thin diamond films. The seeding process consisted of immersing the optical fibre twice for $1 \mathrm{~min}$ in the suspension. The first suspension was prepared in two steps. First, $1 \mathrm{~g}$ of solid PVA (average molar mass - $18000 \mathrm{~g} \mathrm{~mol}^{-1}$ ) was suspended in $99 \mathrm{~g}$ of DMSO at temperature of $80^{\circ} \mathrm{C}$, forming $1 \% \mathrm{w} / \mathrm{w}$ solution. Then, after cooling to room temperature the diamond suspension (DMSO - $0.5 \% \mathrm{w} / \mathrm{w})$ was added dropwise.
The second suspension was prepared simply by diluting $5 \% \mathrm{w} / \mathrm{w}$ DMSO suspension with ethyl alcohol. To get $0.3 \mathrm{~g} / 1$ concentration $6 \mathrm{ml}$ of DMSO suspension was diluted to 11 by alcohol.

Seeding process repeatability was achieved by automatic dip-coating mechanism. The reference Si wafers were spin-coated using the same procedure. All the deposition parameters for each sample ale listed in Table.

TABLE

The set of the fibre samples together with applied deposition and seeding parameters.

\begin{tabular}{c|c|c|c|c|c}
\hline \hline Sample & Substrate & $\begin{array}{c}\text { Gas } \\
\text { flow } \\
{[\mathrm{sccm}]}\end{array}$ & $\begin{array}{c}\mathrm{CH}_{4} / \mathrm{H}_{2} \\
{[\%]}\end{array}$ & $\begin{array}{c}\text { Seeding } \\
\text { suspension }\end{array}$ & $\begin{array}{c}\text { Time } \\
{[\mathrm{min}]}\end{array}$ \\
\hline Fibre1 & fibre & 300 & $4 \%$ & DND in ethanol & 60 \\
Fibre2 & fibre & 300 & $4 \%$ & DND in PVA/DMSO & 60 \\
Si1 & $p$-Si & 300 & $4 \%$ & DND in ethanol & 60 \\
Si2 & $p$-Si & 300 & $4 \%$ & DND in PVA/DMSO & 60
\end{tabular}

\subsection{Nanocrystalline diamond growth}

The diamond films were synthesised using MW PE CVD system (SEKI Technotron AX6200S, Japan). Optical fibres and reference $\mathrm{Si}$ wafers were placed in CVD chamber on a molybdenum stage.

The base pressure (before growth) in the vacuum chamber was $10^{-4}$ Torr. The working pressure was kept at 50 Torr. Methane $\left(\mathrm{CH}_{4}\right)$ and hydrogen $\left(\mathrm{H}_{2}\right)$ were used as the precursor gases, and the flow rates of $\mathrm{CH}_{4}$ and $\mathrm{H}_{2}$ were $12 \mathrm{sccm}$ and $300 \mathrm{sccm}$, respectively. During the process the molybdenum stage was heated up only to $300{ }^{\circ} \mathrm{C}$ by induction heater and controlled by thermocouple. The plasma was generated with microwave radiation $(2.45 \mathrm{GHz}) 1300 \mathrm{~W}$, which is efficient for diamond synthesis [36-38]. Microwaves affect the stage temperature and increase it to $\approx 480{ }^{\circ} \mathrm{C}$. The deposition time was kept at $60 \mathrm{~min}$.

After growth process the substrate temperature was slowly reduced $\left(2{ }^{\circ} \mathrm{C} \mathrm{min}^{-1}\right)$ down to room temperature. The temperature was adjusted by simultaneous lowering of the microwave power and current of induction heater.

\subsection{Optical analytical methods and advanced surface analysis techniques}

The morphology studies were performed by the scanning electron microscope (S-3400N, Hitachi, Japan). Variable pressure SEM mode (VP-SEM) was used, which allows for measuring the sample morphology regardless on its electrical conductivity. Secondary electrons mode has been applied with $20 \mathrm{kV}$ accelerating voltage.

Optical power loss in optical fibres was investigated using home-made setup (Fig. 1). Efficient coupling of a laser beam into a single-mode optical fibre was arranged with the single-mode fibre coupler (Newport, F916, USA). Setup consisted of $\mathrm{He}-\mathrm{Ne}$ laser source with a wavelength of $(632.8 \mathrm{~nm})$ and, an analogue handheld 
optical laser power-meter console (Thorlabs, PM100A, USA) with standard photodiode power detector (Thorlabs, S120VC, USA).

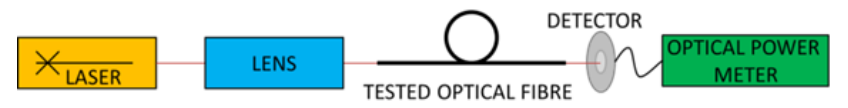

Fig. 1. Scheme of optical power loss setup used for measurement of optical fibres.

Spectroscopic ellipsometry (SE) investigations were carried out with a phase-modulated ellipsometer JobinYvon UVISEL (HORIBA Jobin-Yvon Inc., Edison, USA). The investigated wavelength range was 260 to $830 \mathrm{~nm}$. The experiments were performed at room temperature using an angle of incidence fixed at $70^{\circ}$. Ellipsometric fitting was based on a four-phase optical model (air/surface roughness film (SRL)/diamond/Siwafer). The dispersion of Si (100) was taken from the database [39]. The dielectric function of the SRL was estimated using the Bruggeman effective medium approximation (EMA) [40].

The diamond film has been here assumed to be an isotropic, homogeneous material and its dispersion was fitted to the Tauc-Lorentz (TL) oscillator model. This model has been used recently for amorphous semiconductors by Logothetidis et al. [41, 42]. Such materials exhibit a peculiarity due to the presence of two separated contributions of inter-band electronic transition related to $s p^{2}$ and $s p^{3}$ bonded carbon [43]. The parameters of the TL model were fitted for each of the analysed films. Finally, the assumed optical model was fitted to the experimental data using the non-linear Levenberg-Marquardt regression method for mean-square error minimization (MSE) [44]. As a result of SE analysis, the thickness and optical constants, i.e. refractive index $n(\lambda)$ and extinction coefficient $k(\lambda)$ were obtained.

The molecular composition of the films was studied by means of the Raman spectroscopy using the Raman confocal microscope (Horiba LabRAM ARAMIS, Japan). Spectra were recorded in a range of $200-3500 \mathrm{~cm}^{-1}$ with an integration time of $5 \mathrm{~s}$ (10 averages), using a $532 \mathrm{~nm}$ diode pumped solid state (DPSS) laser in combination with a $50 \times$ objective magnification $(\mathrm{NA}=0.5)$ and $50 \mu \mathrm{m}$ confocal aperture.

\section{Results}

3.1. Evolution of surface morphology and molecular structure along the coated optical fibre

Figure 2 shows SEM micrographs of diamond morphology taken on the side surface of deposited fibre. A significant difference in the morphology related to growth of nanodiamond film on optical fibre deposition on different seeding suspension can be observed. Sample Fibre2 promotes the film growth in the initial stage of process more intensively in comparison to the sample Fibre1. On Fibre1 bigger crystals and discontinuous film structure were observed. It was caused by less nanodiamond particles on optical fibres after dip-coating seeding. The use of PVA/DMSO seeding on Fibre2 results in continuous, homogeneous thin diamond films. Moreover, in both samples it can be observed that the size as well as structure of optical fibre was not defected.
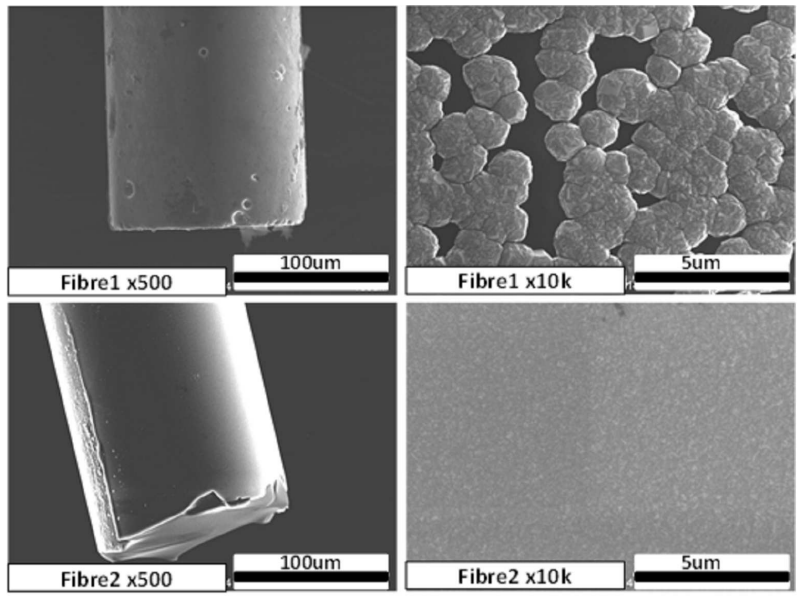

Fig. 2. SEM images of diamond films produced with different seeding suspensions: DND dispersed in ethanol (Fibre1) and DND dispersed in PVA/DMSO (Fibre2). Resolution $\times 500$ and $\times 10000$ micrographs in SE mode, under $20 \mathrm{kV}$ accelerating voltage.

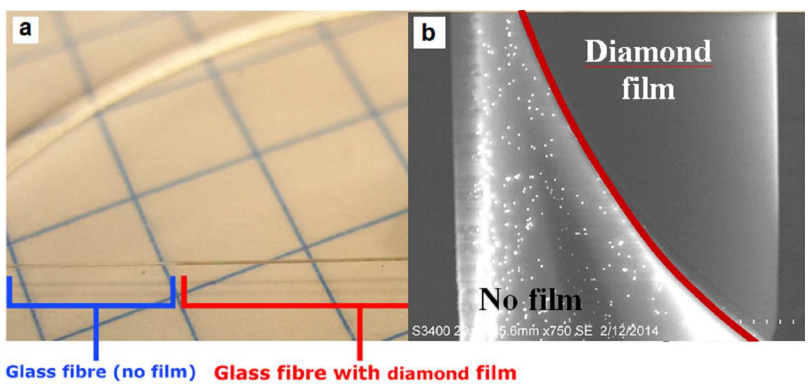

Fig. 3. Photo image (a) and SEM image (b) of selectively covered optical fibre with diamond film.

Figure 3 shows the photo and SEM images of selectively seeded optical fibre. The partially coated fibre surface allows for fusion splicing to another optical fibre (blue part in Fig. 3a). Boundary between fused silica optical fibre surface (dotted white) and diamond coated fibre (grey) can be observed in Fig. 3b.

Figure 4 shows the Raman spectra of diamond film deposited on the optical fibre with two types of dip-coating seeding: dispersed of DND in PVA with DMSO (a) and the DND suspension in ethyl alcohol (b).

Comparison of the Raman spectra of films deposited with different types of suspensions, shows that the spectra are comparable and could be assigned to high quality polycrystalline CVD diamond. Both spectra have strong band at $1332 \mathrm{~cm}^{-1}[39,40]$, which is characteristic line of 


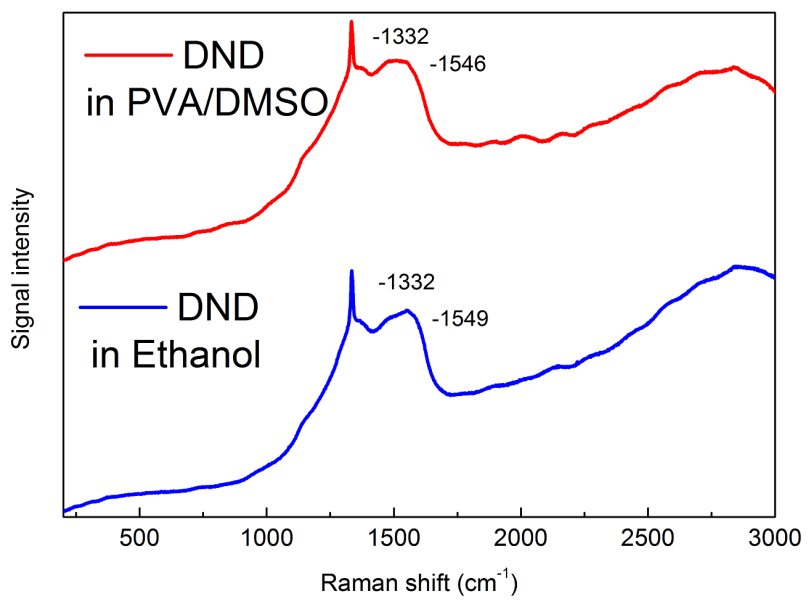

Fig. 4. Raman spectra of the diamond-coated fibre samples treated with two different suspensions: DND dispersed ethanol (Fibre1) and DND dispersed in PVA/DMSO (Fibre2) used to seeding nanodiamond.

diamond. Its presence in both spectra confirms that the diamond can grow with both types of seeding. In addition, one broad band centred at $1546 \mathrm{~cm}^{-1}$ (assigned to amorphous $s p^{2}$ phase and known as "G" band $\left.[45,46]\right)$ is observed in both spectra. Ratio of these bands is similar in both spectra.

\subsection{Optical power loss in diamond-coated fibre}

Optical power loss in diamond-coated fibre was investigated to test waveguiding properties of microwave plasma treated glass fibres. Moreover, CVD diamond deposition at $475{ }^{\circ} \mathrm{C}$ could influence on the fibre core structure.

Results of optical power loss of NCD-coated optical fibres were normalized to power loss of uncoated fibre. Optical power loss of Fibre1 and Fibre2 was equal to 0.85 and 0.92 , respectively. Obtained values for diamond coated fibres differ slightly. Process of nucleation either CVD diamond growth process do not damage waveguiding properties of optical fibres. Diamond coating process affects stiffness of the optical fibres due to properties of polycrystalline diamond films. However, this is not crucial parameter influencing transmittance level. The slightly smaller optical power losses were obtained for the sample Fibre2 that is corresponding to the best quality of diamond films. Transmittance differences between samples arise from properties of diamond films like grain size, film homogeneity or roughness. Presented results are comparable with Marcuse et al. losses level obtained by the misalignment of two fibers joined in a splice [47].

\subsection{Variations of optical properties of nanocrystalline diamond films}

Due to difficulty of making ellipsometric measurements directly on the curved surface of optical fibres, the measurements were instead performed on the reference samples deposited on Si wafers. The applied fitting procedure gives accurate values of film thicknesses, roughness and dispersion of refractive index and extinction coefficient.

Changes of the refractive index and extinction coefficient of the NCD films are presented in Fig. 5. The optical constants variation shows that high refractive index in the range of 2.3-2.4 was achieved for NCD films deposited on optical fibres pre-treated by DND dispersed in PVA/DMSO suspension. The lower refractive index in the range of 2.1-2.3 was achieved for NCD film deposited on fibres previously coated by DND dispersed in ethanol. $\mathrm{Hu}$ et al. [48] reported the variability of $n$ between 2.31 and 2.34 for NCD films, while Gupta et al. [49] determined the $n$ values between 1.7 and 2.1 for MCD films at $\lambda=632 \mathrm{~nm}$. The lower $n$ values (compared to SCD) indicated lower physical density of the films [50, 51].

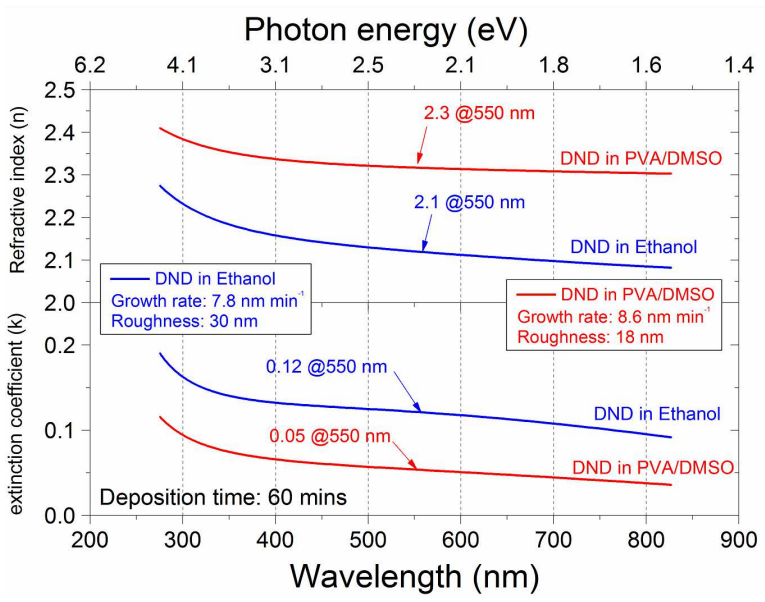

Fig. 5. Dispersion of $\mathrm{n}$ and $\mathrm{k}$ for diamond films deposition using different seeding suspensions: DND dispersed in ethanol (Si1) and DND dispersed in PVA/DMSO (Si2).

The $k$ values obtained in this study reached values below 0.05 and 0.12 at $550 \mathrm{~nm}$ (respectively at sample Si1 and Si2). For Si2 sample it reaches 0.15 at $300 \mathrm{~nm}$ and decreases to values below 0.05 over the $800 \mathrm{~nm}$. Deposited NCD films exhibit high transparency for longer wavelengths range (UV-NIR).

Nevertheless, samples deposited on fibres modified by DND dispersed in PVA/DMSO present reasonable higher refractive index and lower absorption comparing to that previously modified by DND dispersed in ethanol.

Since refractive index is correlated with material density $[51,52]$, these results suggest that the density of samples deposited on fibres pre-treatment by DND dispersed in PVA/DMSO are higher than that of sample deposited on DND dispersed in ethanol. The 60 min growth results in NCD film thicknesses of $472 \mathrm{~nm}$ and $517 \mathrm{~nm}$ for DND dispersed in ethanol and DND dispersed in PVA/DMSO pre-treated sample, respectively. The growth rates of deposited NCD film including surface roughness (SRL) are summarized in Fig. 5. All the deposited diamond films show normal dispersion. The optical constants 
decrease with increasing wavelength, exhibiting a typical behaviour near the band gap of electronic transition. Moreover, the values of optical constants for all NCD films are not shifted relating to each other over the wavelength range.

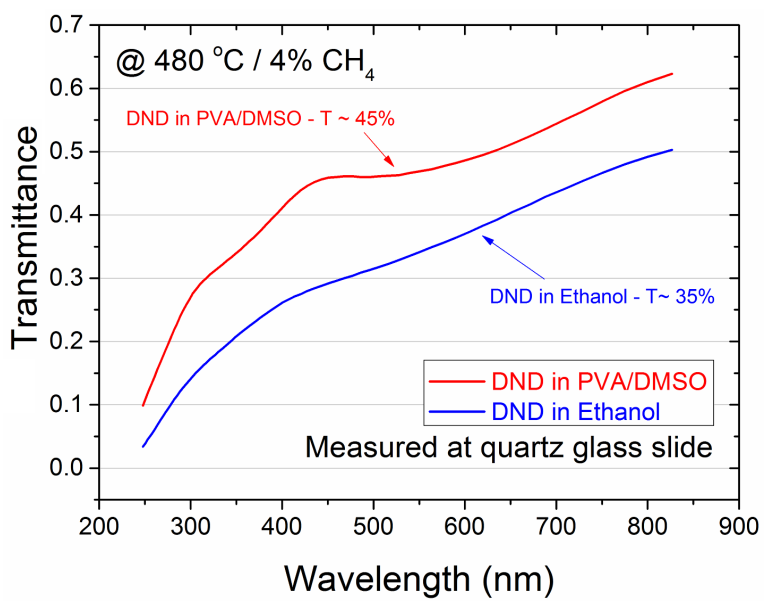

Fig. 6. Transmittance of diamond film measured at quartz glass slide.

The transmittance of NCD films grown on quartz glass slide substrates were shown in Fig. 6. It could be observed that the transmittance values are below $10 \%$ at $300 \mathrm{~nm}$. They increase to values above $60 \%$ over the $800 \mathrm{~nm}$. The transmittance values below $45 \%$ at $550 \mathrm{~nm}$ were achieved for NCD films grown on the glass modified by DND dispersed in PVA/DMSO suspension. The lower transmittance values of $35 \%$ at $550 \mathrm{~nm}$ were achieved for NCD film grown on quartz glass slide covered by DND dispersed in ethanol.

\section{Conclusions}

We have demonstrated that NCD films on fused silica fibres could be better improved by dip coating seeding in PVA/DMSO nanodiamond suspension than by standard ethanol nanodiamond suspension. Although presence of the strong diamond line $\left(1332 \mathrm{~cm}^{-1}\right)$ in both Raman spectra confirms that both suspensions induces the efficient nucleation of diamond films having high $s p^{3}$ content, the applied PVA/DMSO suspension has important advantages in comparison to ethanol.

Contrary to single point Raman spectra, SEM imaging shows that the use of PVA admixture to nanodiamond suspension improves seeding efficiency on the fused silica fibres resulting in continuous and uniform nanocrystalline diamond coating.

Ethanol-based nanodiamond suspension results in law seeding density exhibiting defects in surface continuity and relatively high grain size causing light scattering (SEM microimage of Fibre1).

The optical constants variation shows that high refractive index of 2.4 at $550 \mathrm{~nm}$ was achieved for NCD films deposited using PVA/DMSO. Nevertheless, the ethanolbased DND suspension results in refractive index limited to 2.1 at $550 \mathrm{~nm}$.

The samples deposited using PVA/DMSO present reasonable lower absorption measures as extinction coefficient decreases comparing to the DND dispersed in ethanol. Moreover, the transmittance achieves values of $45 \%$ and $35 \%$ at $550 \mathrm{~nm}$ for NCD films deposited using seeding in PVA/DMSO suspension and ethanolbased suspension, respectively. Uniform and continuous high refractive index diamond coatings could be applied as a protective and sensitivity enhancing coverage of optical fibre sensors. Due to extraordinary properties of diamond, which include high chemical and mechanical resistance, such films are highly desired for optical sensing purposes.

\section{Acknowledgments}

The authors gratefully acknowledge financial support from the Polish National Science Centre (NCN) under the Grant No. 2011/03/D/ST7/03541. The DS funds of Faculty of Electronics, Telecommunications and Informatics of the Gdańsk University of Technology are also acknowledged.

\section{References}

[1] B. Lee, Opt. Fiber Technol. 9, 57 (2003).

[2] O.S. Wolfbeis, Anal. Chem. 76, 3269 (2004).

[3] Y. Liu, Z. Guo, Y. Zhang, K.S. Chiang, X. Dong, Electron. Lett. 36, 564 (2000).

[4] Y. Zhu, A. Wang, IEEE Photon. Technol. Lett. 17, 447 (2005).

[5] A. Mehta, W. Mohammed, E.G. Johnson, IEEE Photon. Technol. Lett. 15, 1129 (2003).

[6] P.W. Baumeister, Optical Coating Technology, SPIE Publ., Bellingham, WA 2004.

[7] M. Benounis, N. Jaffrezic-Renault, Sens. Actuat. B Chem. 100, 1 (2004).

[8] S. Aoqui, K. Ebihara, Y. Yamagata, Carbon 36, 591 (1998).

[9] J. Stotter, S. Haymond, J.K. Zak, Y. Show, Z. Cvackova, G.M. Swain, Interface 12, 33 (2003).

[10] R. Bogdanowicz, Metrol. Measur. Syst. 21, 685 (2014).

[11] M. Panizza, G. Cerisola, Electrochim. Acta 51, 191 (2005).

[12] A.V. Sukhadolau, E.V. Ivakin, V.G. Ralchenko, A.V. Khomich, A.V. Vlasov, A.F. Popovich, Diam. Relat. Mater. 14, 589 (2005).

[13] M. Amaral, A.G. Dias, P.S. Gomes, M.A. Lopes, R.F. Silva, J.D. Santos, M.H. Fernandes, J. Biomed. Mater. Res. Part A 87A, 91 (2008).

[14] P. Bajaj, D. Akin, A. Gupta, D. Sherman, B. Shi, O. Auciello, R. Bashir, Biomed. Microdev. 9, 787 (2007). 
[15] J. Philip, P. Hess, T. Feygelson, J.E. Butler, S. Chattopadhyay, K.H. Chen, L.C. Chen, J. Appl. Phys. 93, 2164 (2003).

[16] P. Bergonzo, A. Brambilla, D. Tromson, C. Mer, B. Guizard, R.D. Marshall, F. Foulon, Nucl. Instrum. Methods Phys. Res. A 476, 694 (2002).

[17] H.-J. Lee, H. Jeon, W.-S. Lee, J. Phys. Chem. C 116, 9180 (2012).

[18] D.M. Gruen, Ann. Rev. Mater. Sci. 29, 211 (1999).

[19] Y. Lifshitz, C.H. Lee, Y. Wu, W.J. Zhang, I. Bello, S.T. Lee, Appl. Phys. Lett. 88, 243114 (2006).

[20] J. Asmussen, D.K. Reinhard, Diamond Films Handbook, CRC Press, New York 2002.

[21] O. Shenderova, S. Hens, G. McGuire, Diam. Relat. Mater. 19, 260 (2010).

[22] O.A. Williams, O. Douhéret, M. Daenen, K. Haenen, E. Osawa, M. Takahashi, Chem. Phys. Lett. 445 , 255 (2007).

[23] A. Kromka, O. Babchenko, H. Kozak, K. Hruska, B. Rezek, M. Ledinsky, J. Potmesil, M. Michalka, M. Vanecek, Diam. Relat. Mater. 18, 734 (2009).

[24] M. Tsigkourakos, T. Hantschel, S.D. Janssens, K. Haenen, W. Vandervorst, Phys. Status Solidi A, 209, 1659 (2012).

[25] E.I. Givargizov, V.V. Zhiraov, A.V. Kuznetsov, P.S. Plekhanov, Mater. Lett.18, 61 (1993).

[26] Y. Lifshitz, C.H. Lee, Y. Wu, W.J. Zhang, I. Bello, S.T. Lee, Appl. Phys. Lett. 88, 243114 (2006).

[27] R. Bogdanowicz, M. Śmietana, M. Gnyba, M. Ficek, V. Straňák, Ł. Goluński, J. Ryl, Phys. Status Solidi A 210, 1991 (2013).

[28] M. Śmietana, M. Dudek, M. Koba, B. Michalak, Phys. Status Solidi A 210, 2100 (2013).

[29] P.W. May, C.A. Rego, M.N.R. Ashfold, K.N. Rosser, G. Lu, T.D. Walsh, P.G. Partridge, Diam. Relat. Mater. 4, 794 (1995).

[30] J.R. Rabeau, S.T. Huntington, A.D. Greentree, S. Prawer, Appl. Phys. Lett. 86, 134104 (2005).

[31] R. Bogdanowicz, M. Śmietana, M. Gnyba, Ł. Gołunski, J. Ryl, M. Gardas, Appl. Phys. A 116, 1927 (2014).

[32] E. Scorsone, S. Saada, J.C. Arnault, P. Bergonzo, J. Appl. Phys. 106, 014908 (2009).
[33] R. Kiran, E. Scorsone, P. Mailley, P. Bergonzo, Anal. Chem. 84, 10207 (2012).

[34] R.F. Davis, Diamond Films and Coatings Development, Properties, and Applications, Noyes Pub, Park Ridge, NJ 1993.

[35] M. Kraszewski, R. Bogdanowicz, Metrol. Measur. Syst. 20, 543 (2013).

[36] R. Bogdanowicz, M. Gnyba, P. Wroczyński, J. Phys IV (Proc.) 137, 57 (2006).

[37] R. Bogdanowicz, M. Gnyba, P. Wroczyński, B.B. Kosmowski, J. Optoelectron Adv. Mater. 12, 1660 (2010).

[38] R. Bogdanowicz, Acta Phys. Pol. A 114, A33 (2008).

[39] Handbook of Optical Constants of Solids, Vol. 2, 1st ed., Ed. E.D. Palik, Academic Press, 1991.

[40] H.G. Tompkins, A User's Guide to Ellipsometry, Academic Press, New York 1992.

[41] M. Gioti, D. Papadimitriou, S. Logothetidis, Diam. Relat. Mater. 9, 741 (2000).

[42] S. Logothetidis, M. Gioti, P. Patsalas, C. Charitidis, Carbon 37, 765 (1999).

[43] G.E. Jellison, F.A. Modine, Appl. Phys. Lett. 69 371 (1996).

[44] H. Tompkins, E.A. Irene, in: Handbook of Ellipsometry, Eds. H. Tompkins, E.A. Irene, 1st ed., William Andrew, Norwich 2006, p. 237.

[45] D.S. Knight, W.B. White, J. Mater. Res. 4, 385 (1989).

[46] M. Gnyba, in: Presented at the XX IMEKO World Congress, Busan (Republic of Korea), 2012, p. 38. Retrieved from http://www.imeko.org/publications/wc2012/IMEKO-WC-2012-TC2-P2.pdf.

[47] D. Marcuse, Bell Syst. Techn. J. 56, 703 (1977).

[48] Z.G. Hu, P. Prunici, P. Hess, K.H. Chen, J. Mater. Sci. Mater. Electron. 18, 37 (2007).

[49] S. Gupta, B.R. Weiner, G. Morell, J. Appl. Phys. 90, 1280 (2001).

[50] J. Robertson, Mater. Sci. Eng. R Rep 37, 129 (2002).

[51] G. Davies, Properties and Growth of Diamond, INSPEC, the Institution of Electrical Engineers, 1994.

[52] J. Robertson, Mater. Sci. Eng. R Rep. 37, 129 (2002). 